\title{
Combined Pressure-Driven and Electroosmotic Flow of Casson Fluid Through a Slit Microchannel
}

\author{
Chiu-On $\mathrm{Ng}^{1}$ \\ Department of Mechanical Engineering, The University of Hong Kong, \\ Pokfulam Road, Hong Kong
}

April 24, 2013

\begin{abstract}
This study aims to develop analytical solutions for steady electroosmotic (EO) flow of a viscoplastic material, namely Casson fluid, through a parallel-plate microchannel. The flow is driven by electric as well as pressure forcings. A very thin electric double layer is assumed, and the Debye-Hückel approximation is used. The Casson yield stress makes the present problem distinct from existing studies on EO flow of other kinds of non-Newtonian such as power-law fluids. A first step of the analysis is to locate the yield surface, which divides the flow section into sheared and unsheared regions, where the stress is larger and smaller in magnitude than the yield stress, respectively. Different combinations of the electric and pressure forcings can lead to different types of distribution of stress relative to the yield stress. In this study, integrals of the nonlinear coupling terms of the two forcings are analytically expressed by uniformly valid approximations derived using the boundary-layer theory. It is shown that even a small value of the Casson yield stress, characteristic of that of blood, can considerably reduce the rate of flow of the fluid through a microchannel by electroosmosis. The decreasing effect of the yield stress on the flow is intensified by the presence of a pressure gradient, whether favorable or adverse.

Keywords: Electroosmotic flow; electric double layer; Casson fluid; viscoplastic yield stress.
\end{abstract}

\footnotetext{
${ }^{1}$ Tel: (852) 2859 2622; Fax: (852) 28585415 ; E-mail address: cong@hku.hk (C.-O. Ng).
} 


\section{Introduction}

The rheology of fluid is an important consideration in microflows: Newtonian as well as non-Newtonian fluids need to be considered in engineering flows through microdevices [1]. In these small devices, polymeric solutions are often used as the media for DNA and protein separation [2]. Colloidal systems involving blood are also often implemented on a microscale. A Newtonian model will fall short in describing the rheology of these systems.

The need to understand the effect of non-Newtonian rheology in microflows has spurred a growing interest in this topic in recent years. Flow through a microchannel is commonly driven by a pressure gradient, an electric field, or a combination of both. Flow driven by electric field works on the basis of electroosmosis, and is therefore known as electroosmotic (EO) flow. A body force that drags fluid into motion arises when the electric field interacts with the unbalanced charge in an electric double layer (EDL), which is formed at the contact interface of an electrolyte and a solid surface.

While pressure-driven non-Newtonian flow has long been studied, the EO flow of a non-Newtonian fluid was not theoretically investigated until recently. Motivated to study biofluids in micro-systems, Das and Chakraborty [3], and Zimmerman et al. [4] were among the first to present theoretical work on non-Newtonian electrokinetic flow and transport in microchannels. The non-Newtonian model adopted by Das and Chakraborty [3] is the power-law model, while that by Zimmerman et al. [4] is the Carreau model. The power-law (also known as Ostwald-de Waele) model is one of the simplest models to describe nonlinear viscous behaviors. It is a two-parameter model, where the shear-thinning or shear-thickening behaviors can be conveniently represented by the flow index being less than or larger than 1, respectively. For sufficiently simple geometry and in the absence of other forcings, it is possible to determine EO flow of a power-law fluid analytically.

EO flow of a power-law fluid in microchannels of various shapes (single surface, parallel-plate, rectangular, cylindrical, or annular) has since then been intensively studied. For relatively simple flows (e.g., one-dimensional flow, no pressure forcing, linearized Poisson-Boltzmann equation), analytical or semi-analytical solutions (exact or approx- 
imate) have been obtained by Chakraborty [5], Berli and Olivares [6], Zhao et al. [7], Olivares et al. [8], Zhao and Yang [9-11], Berli [12], Vasu and De [13], and Sadeghi et al. [14]. More complicated flows (e.g., two-dimensional flow, unsteady flow, nonlinear coupling of the EO and pressure forcings, nonlinear Poisson-Boltzmann equation) can only be solved or simulated numerically. Such numerical studies have been performed by Bharti et al. [15], Tang et al. [16], Vasu and De [17], Babaie et al. [18], Hadigol et al. [19], Cho et al. [20,21], Deng et al. [22], Shamshiri et al. [23], and Vakili et al. [24].

Because of its simplicity, the power-law model has been the most chosen model to describe non-Newtonian rheology in EO flow through microchannels. Much fewer existing studies are based on other models. The Bingham, Herschel-Bulkley, and Carreau models have been selected only in a limited number of studies (e.g., Berli and Olivares [6], Tang et al. [25], Zhao and Yang [26]).

The present study aims to look into flow of a particular kind of viscoplastic material, viz. Casson fluid, under the combined action of electrokinetics and pressure gradient in a parallel-plate microchannel. Polymeric solutions and colloidal suspensions are often classified as viscoplastic materials. They exhibit a yield stress, a critical value of stress below which the materials do not flow or flow like a rigid body. Most viscoplastic materials can be described by the Bingham, Casson, or Herschel-Bulkley models [27]. Electrokinetic driven flow of Casson fluids in microcapillaries has been studied by Liu and Yang [28] and Liu et al. [29], who applied a two-phase Newtonian/non-Newtonian model to describe blood flow.

The model proposed by Casson [30] can be generalized into

$$
\mu \dot{\gamma}= \begin{cases}{\left[1-{\sqrt{\tau_{0} /|\tau|}}^{2} \tau\right.} & \text { for }|\tau|>\tau_{0} \\ 0 & \text { for }|\tau| \leq \tau_{0}\end{cases}
$$

where $\tau$ is the stress tensor, $\dot{\gamma}=\nabla \mathbf{u}+(\nabla \mathbf{u})^{T}$ is the rate of deformation tensor, $\mu$ is the plastic viscosity, $\tau_{0} \geq 0$ is the Casson yield stress, and

$$
|\tau|=\sqrt{\frac{1}{2} \tau: \tau}
$$

is the magnitude of the stress. The Casson model reduces to the Newtonian model when the yield stress vanishes $\tau_{0}=0$, by which $\mu$ becomes the Newtonian viscosity. Even with 
a non-zero yield stress, a Casson fluid will asymptotically behave like a Newtonian fluid as the stress increases to a level much higher than the yield stress. The viscosity $\mu$ is also known as the limiting Newtonian (high-shear-rate) viscosity.

The Casson model was originally developed for pigment-oil suspensions of the printing ink. It is now commonly used to describe the rheology of blood [31,32]. The yield stress of blood is a strong function of the volume fraction of red blood cells, or the hematocrit. At hematocrit of $40 \%$, the yield stress for normal blood is typically 0.04 dynes $/ \mathrm{cm}^{2}$ or $4 \mathrm{mPa}$ [32]. Such a small value of yield stress is often regarded as physiologically insignificant for blood flow in large vessels. The significance of the Casson yield stress, however, remains to be determined for EO flow in microchannels. This has motivated the present study. The behaviors of a Casson fluid under EO pumping in a microchannel are yet to be fully understood.

A complication arises in the analysis of flow of a yield-stress fluid. It needs to divide the flow domain into regions where the stress is smaller or larger in magnitude than the yield stress. The two regions are accordingly called unsheared (or unyielded) and sheared (or yielded) regions. The location of the yield surface, which is the interface between the two regions, is in general not known a priori, and has to be found as part of the final solution. The problem then becomes highly nonlinear, and can only be solved numerically. To study the physics as analytical as possible, we shall consider in this study strictly one-dimensional flow in a uniform channel, for which the stress distribution is known prior to finding the velocity. With a known stress distribution, the position of the yield surface can be readily determined. The velocity can then be found straightforwardly.

Another complication arises when the flow is simultaneously driven by two forcings. Owing to nonlinear rheology, linear superposition of solutions due to individual forcings is not applicable. A nonlinear coupling term of the two forcings is bound to appear when the Cauchy momentum equation is solved for the velocity. As a result, the velocity may not be analytically expressed, unless some approximations are made (e.g., Zhao and Yang [9]).

In the present problem, both pressure and electric forcings are considered, and they 
can independently affect the stress distribution. Different combinations of the two forcings will lead to qualitatively different stress distributions, thereby different types of the partitioning into sheared and unsheared regions. We shall first identify all the possible cases of stress distribution, and then derive expressions for the velocity in each case. To obtain analytical expressions for the velocity, we shall use the boundary-layer theory to approximate the integral of the nonlinear coupling term. If the electric double layer (EDL) is very thin compared with the channel height, the stress distribution will have two components. Across the entire channel section is a slowly varying component due to the pressure forcing, while near the wall is a rapidly varying component due to the electric forcing. By virtue of this boundary-layer structure, an asymptotically uniform approximation can be deduced for the integral.

In the modeling of flow of non-Newtonian polymeric solutions in microchannels, a two-phase distribution of rheology is often considered: near the wall is a thin layer which is depleted of polymer molecules and is hence Newtonian, while outside this layer the fluid is non-Newtonian. This two-phase configuration also accounts for the well-known Fahraeus-Lindqvist effect in blood flow in a capillary: the apparent viscosity of blood decreases as the tube diameter decreases. This is due to the fact that adjacent to the capillary wall is a plasma skimming layer, which is depleted of red blood cells and is hence a region of reduced viscosity. Such a near-wall depletion effect has been incorporated in the EO flow models by, among others, Zimmerman et al. [4], Berli and Olivares [6], Olivares et al. [8], Berli [12], Liu and Yang [28], and Liu et al. [29]. In the present problem, we shall, however, not consider the depletion layer. We simply consider the EO flow of a one-phase fluid that is homogeneously non-Newtonian. This will enable us to look into the coupling effect of the electrokinetic forcing and the non-Newtonian rheology, as has been done by Babaie et al. [18] and Hadigol et al. [19]. We leave it to a future study to extend the problem to a two-phase model.

Our problem is further described in Section 2, where assumptions are stated as the problem is mathematically formulated. The various possible cases of stress distributions are then described in Section 3. For each case of stress distribution, the position(s) of the yield surface(s) and the velocity are analytically derived. We shall then examine in Section 4, through some numerical results, the effect of the yield stress on the flow rate 
as a function of the pressure gradient. Velocity profiles are also presented for illustration of the yield stress effects. Some concluding remarks are finally given in Section 5 .

\section{Combined pressure-driven and electroosmotic flow}

We consider steady flow through a microchannel bounded by two parallel plates, which are uniformly charged with a zeta potential $\zeta$, and at a distance $2 h$ apart. Figure 1 shows a definition sketch of our problem, where $(x, y)$ are the streamwise and transverse coordinates, respectively. The channel is filled with a non-Newtonian liquid electrolyte, which can be modeled as a Casson fluid. Because of symmetry of the flow about the mid-plane, it suffices for us to consider only the lower half of the channel $0 \leq y \leq h$.

The flow is simultaneously forced by a constant electric field $E_{x}$ and a constant pressure gradient $P_{x}=-d p / d x$, both purely in the $x$-direction. When fully developed, the flow is strictly one dimensional, and is governed by the Cauchy momentum equation as follows:

$$
P_{x}+\frac{d \tau}{d y}+\rho_{e} E_{x}=0
$$

where $\tau=\tau_{x y}(y)$ is the shear stress, and $\rho_{e}(y)$ is the free charge density in the electric double layer (EDL) of the channel wall. Here, the pressure gradient and the electric field can be both externally applied (and thereby independent of each other), or one is applied while the other is induced (e.g., streaming potential in pressure-driven flow, or electroosmotic pressure induced in EO flow in a closed channel). For generality, we shall treat them as two independent forcings in our following analysis.

The electric potential $\psi(y)$, measured relative to the bulk solution phase, where $\psi$ is zero, is related to the charge density $\rho_{e}$ by the Poisson equation (which simplifies in one dimension into)

$$
\frac{d^{2} \psi}{d y^{2}}=-\frac{\rho_{e}}{\epsilon},
$$

where $\epsilon$ is the dielectric permittivity of the liquid electrolyte.

With Eq. (4), Eq. (3) can be integrated to give the stress distribution

$$
\tau(y)=P_{x}(h-y)+\epsilon E_{x} \frac{d \psi}{d y}
$$


where the symmetry boundary conditions $\tau=0$ and $d \psi / d y=0$ at $y=h$ have been used. This stress distribution is valid for any type of generalized Newtonian fluid.

Consider a symmetrical electrolyte of valence $z$ and a Boltzmann distribution of the electrolyte ions in the EDL, the charge density is given by

$$
\rho_{e}=-2 z e n \sinh \left(\frac{z e \psi}{k_{B} T}\right),
$$

where $e$ is the fundamental charge, $n$ is the bulk concentration, $k_{B}$ is Boltzmann's constant, and $T$ is the absolute temperature. Putting Eq. (6) into Eq. (4) will form the well-known Poisson-Boltzmann equation. On further assuming small potentials, $z e \psi / k_{B} T \ll 1$, the Poisson-Boltzmann equation can be linearized to

$$
\frac{d^{2} \psi}{d y^{2}}=\kappa^{2} \psi
$$

where $\kappa=\left(2 z^{2} e^{2} n / \epsilon k_{B} T\right)^{1 / 2}$ is termed the Debye parameter, the inverse of which is the Debye shielding length of the EDL. The linearization of the Poisson-Boltzmann equation is also known as the Debye-Hückel approximation. In view of the fact that the Debye length is usually of the order of tens of nanometers (for a buffer concentration larger than $1 \mathrm{mM}$ ), while the height of a microchannel is usually of the order of microns, it is reasonable to further assume that the EDL is much thinner than the channel height, or $\kappa h \gg 1$. Therefore, the electric potential is essentially zero in the core of the channel. With the boundary conditions $\psi(0)=\zeta$ and $\psi(h)=0$, where $\zeta$ is the wall zeta potential, Eq. (7) can be readily solved to give

$$
\psi(y)=\zeta e^{-\kappa y}
$$

Substituting this into Eq. (5), we now obtain the stress distribution as

$$
\tau(y)=P_{x}(h-y)+\tau_{E} e^{-\kappa y} \quad \text { in } 0 \leq y \leq h,
$$

where

$$
\tau_{E}=-\epsilon \kappa \zeta E_{x}
$$

is the wall stress induced by the electric field. Without loss of generality, we shall assume that the electric wall stress $\tau_{E}$ is always positive, while the pressure gradient $P_{x}$ can be positive (favorable), zero, or negative (adverse). 
The liquid solution is assumed to behave like a Casson fluid with a constant yield stress $\tau_{0}$ and constant plastic viscosity $\mu$. Under simple shear, the Casson constitutive Eq. (1) reduces to

$$
\mu \frac{d u}{d y}= \begin{cases}\tau-2 \tau_{0}^{1 / 2} \tau^{1 / 2}+\tau_{0} & \text { for } \tau>\tau_{0} \\ 0 & \text { for }|\tau| \leq \tau_{0} \\ \tau+2 \tau_{0}^{1 / 2}(-\tau)^{1 / 2}-\tau_{0} & \text { for } \tau<-\tau_{0}\end{cases}
$$

One may note that by omitting the terms containing $\tau_{0}^{1 / 2}$ and reversing the sign of $\tau_{0}$, the above equation becomes the Bingham model. As will be shown in the next section, the significance of the present problem is much due to the term $\tau_{0}^{1 / 2}$. Hence, the effects to be found here are specific to a Casson fluid, and may not be manifested in other yield-stress materials like Bingham plastic.

To facilitate discussion, let us introduce the following normalized variables (distinguished by an overhead caret) and dimensionless parameters:

$$
\hat{y}=y / h, \quad \hat{\kappa}=\kappa h, \quad \hat{u}=u /\left(\tau_{E} / \mu \kappa\right), \quad(\hat{\tau}, \alpha, \beta)=\left(\tau, P_{x} h, \tau_{0}\right) / \tau_{E} .
$$

Note that the velocity is normalized by $\tau_{E} / \mu \kappa=-\epsilon \zeta E_{x} / \mu$, which amounts to the Smoluchowski slip velocity for Newtonian fluid. The parameter $\beta$, which is the normalized yield stress, is always non-negative. The other parameter $\alpha$, which represents the normalized pressure gradient, can be positive (favorable pressure gradient), zero, or negative (adverse pressure gradient).

In terms of the normalized variables and dimensionless parameters, Eqs. (9) and (11) can be written as

$$
\begin{gathered}
\hat{\tau}=\alpha(1-\hat{y})+e^{-\hat{\kappa} \hat{y}} \quad \text { in } 0 \leq \hat{y} \leq 1, \\
\frac{d \hat{u}}{d \hat{y}}= \begin{cases}\hat{\kappa}\left[\hat{\tau}-2 \beta^{1 / 2} \hat{\tau}^{1 / 2}+\beta\right] & \text { for } \hat{\tau}>\beta \\
0 & \text { for }|\hat{\tau}| \leq \beta \\
\hat{\kappa}\left[\hat{\tau}+2 \beta^{1 / 2}(-\hat{\tau})^{1 / 2}-\beta\right] & \text { for } \hat{\tau}<-\beta\end{cases}
\end{gathered}
$$

These two equations form the basis of the present study. In the following section, we shall look into various possible stress distributions and deduce the corresponding velocity profiles. From here on, we shall for simplicity drop the overhead carets. 


\section{$3 \quad$ Possible stress and velocity profiles}

It is necessary that, in order to determine the velocity, the flow domain is divided into sheared and unsheared regions, where the stress is larger and smaller in magnitude than the yield stress, respectively. The interface between the two regions, known as the yield surface, along which the stress is equal in magnitude to the yield stress, needs to be located before the flow in individual regions can be determined. In the present one-dimensional problem, for which the stress distribution is known before the velocity is found, it is straightforward to locate the yield surface and to identify the sheared/unsheared regions. Figure 2 shows schematically 6 possible cases. In any case, the fluid will remain static until the maximum stress exceeds the yield stress. In the following sections, we shall provide for each case the condition(s) for fluid in motion pertinent to the case, as well as analytical expressions for the yield height(s), and for the velocity profile.

\subsection{Base case: $\alpha=0,1>\beta$}

In the absence of pressure gradient, the stress distribution reduces to $\tau=\exp (-\kappa y)$, as shown in Fig. 2(a). The shear stress drops sharply from unity at $y=0$ to virtually zero far from the wall. In this case, the maximum stress occurs at the wall, and the condition for the fluid to be set into motion is

$$
1>\beta
$$

There is only one yield surface, located at a height $y_{0}$ (referred to as the yield height) given by $\exp \left(-\kappa y_{0}\right)=\beta$, or

$$
y_{0}=-\kappa^{-1} \ln \beta, \quad \text { where } 0 \leq y_{0} \leq 1
$$

The height of the yield surface is at $y_{0}=0$ when $\beta=1$ (i.e., at the threshold of motion). The height increases monotonically as the yield stress decreases. The limiting case is $y_{0}=1$ when $\beta=0$ (i.e., a vanishing unsheared region at the Newtonian limit). For a finite value of $1>\beta>0$, because of $\kappa \gg 1$, the yield height is very small $y_{0} \ll 1$, or the yield surface is located within the EDL near the wall, as expected. The sheared region 
is $0 \leq y<y_{0}$, within which the velocity satisfying no slip at the wall can be readily found to be

$$
u(y)=1-e^{-\kappa y}-4 \beta^{1 / 2}\left(1-e^{-\kappa y / 2}\right)+\beta \kappa y \quad \text { in } 0 \leq y<y_{0} .
$$

The unsheared region is $y_{0}<y \leq 1$, within which the velocity is uniform; it is hence a plug flow region:

$$
u_{p}=u\left(y_{0}\right)=1-4 \beta^{1 / 2}+\beta(3-\ln \beta) \quad \text { in } y_{0}<y \leq 1
$$

When $\beta=0$, the Newtonian limits $u(y)=1-\exp (-\kappa y)$ and $u_{p}=1$ are recovered.

Since $y_{0} \ll 1$, the plug flow region is nearly as wide as the channel section. Hence, the yield stress will have little effect on the size of the plug flow region, but can have significant effect on the flow itself. It is easy to see that for $1>\beta>0$, the plug flow velocity is always less than 1 . Therefore, the yield stress causes the Smoluchowski velocity to be smaller than the Newtonian limit. Some sample values of $y_{0}$ and $u_{p}$ as functions of $\beta$ are shown in Table 1. Even a normalized yield stress as small as $\beta=0.001$ can decrease the plug flow velocity by more than $10 \%$. This decreasing effect is so significant mainly because of the term $-4 \beta^{1 / 2}$ in Eq. (18). For Newtonian EO flows, the Smoluchowski velocity is often used as a slip boundary condition. Owing to nonlinear rheology, such an approach is no longer valid here.

Table 1: The yield height $y_{0}$ and the plug flow velocity $u_{p}$ as functions of the normalized yield stress $\beta$, as given by Eqs. (16) and (18), where $\kappa=100$.

\begin{tabular}{lll}
\hline$\beta$ & $y_{0}$ & $u_{p}$ \\
\hline 0 & 1 & 1 \\
0.001 & 0.069 & 0.883 \\
0.01 & 0.046 & 0.676 \\
0.1 & 0.023 & 0.265 \\
0.5 & 0.007 & 0.018 \\
\hline
\end{tabular}




\subsection{Case 1: $\alpha>0, \alpha+1>\beta$}

With a favorable pressure gradient, the stress decreases monotonically away from the wall, as shown in Fig. 2(b). Therefore, the fluid will be in motion only when the wall stress exceeds the yield stress:

$$
\alpha+1>\beta
$$

There exists only one yield surface, which is at a height $y_{0}$ given by

$$
\alpha\left(1-y_{0}\right)+e^{-\kappa y_{0}}=\beta
$$

which has to be solved numerically for $y_{0}$. Numerical solution can be sought by iteration with one of the following initial guesses:

$$
y_{0} \approx\left\{\begin{array}{ll}
-\kappa^{-1} \ln (\beta-\alpha) & \text { for } \beta>\alpha \\
-\kappa^{-1} \ln (0.1 \alpha) & \text { for } \beta=\alpha \\
1-\beta / \alpha & \text { for } \beta<\alpha
\end{array} .\right.
$$

The above three sub-cases are referred to as cases 1(a), 1(b) and 1(c), respectively. In the sheared region $0 \leq y<y_{0}$, the velocity satisfying no slip at the wall can be found as follows:

$$
u(y)=\alpha \kappa\left(y-\frac{1}{2} y^{2}\right)+\beta \kappa y+1-e^{-\kappa y}-2 \beta^{1 / 2} \kappa I_{1}(y),
$$

where $I_{1}$ is an integral of the nonlinear coupling term

$$
I_{1}(y)=\int_{0}^{y}\left[\alpha(1-y)+e^{-\kappa y}\right]^{1 / 2} d y
$$

In the unsheared region $y_{0}<y \leq 1$, the plug flow velocity is $u_{p}=u\left(y_{0}\right)$ by continuity of velocity. The velocity is everywhere positive in this case. When $\beta=0$, Eq. (22) reduces to the Newtonian limit $u(y)=\alpha \kappa\left(y-y^{2} / 2\right)+1-\exp (-\kappa y)$, which without the coupling term is good for any $\alpha$. Only in the Newtonian limit can the Smoluchowski velocity, which has a normalized value of unity here, be used as a boundary slip velocity in the case of a very thin EDL $\kappa \gg 1$.

There exists no exact analytical expression for the integral $I_{1}$ in general. In view of the fact that the integrand has a boundary-layer structure, namely rapid variation near $y=0$, a uniformly valid approximation can be derived for this integral. Following the boundary-layer theory, we may approximate the integrand by $\left[\alpha+e^{-\kappa y}\right]^{1 / 2}$ in the 
inner region $\kappa y=O(1)$, and by $[\alpha(1-y)]^{1 / 2}$ in the outer region $\kappa y \gg 1$. For these approximations, we obtain

$$
\begin{aligned}
I_{1}^{\mathrm{in}}(y) & =\int_{0}^{y}\left[\alpha+e^{-\kappa y}\right]^{1 / 2} d y \\
& =\frac{2}{\kappa}\left[\alpha^{1 / 2} \sinh ^{-1}\left(\alpha e^{\kappa y}\right)^{1 / 2}-\left(\alpha+e^{-\kappa y}\right)^{1 / 2}\right]_{0}^{y} \quad \text { for } \kappa y=O(1),
\end{aligned}
$$

where $\left.F\right|_{a} ^{b}$ stands for $F(b)-F(a)$, and

$$
\begin{aligned}
I_{1}^{\text {out }}(y) & =\int^{y}[\alpha(1-y)]^{1 / 2} d y \\
& =-\frac{2}{3} \alpha^{1 / 2}(1-y)^{3 / 2}+C \quad \text { for } \kappa y \gg 1
\end{aligned}
$$

where $C$ is a constant to be determined by asymptotic matching. The above two expressions should match each other in their asymptotic limits $I_{1}^{\text {match }}(y)=I_{1}^{\text {in }}\left(y \gg \kappa^{-1}\right)=$ $I_{1}^{\text {out }}(y \ll 1)$, where

$$
\begin{gathered}
I_{1}^{\text {in }}\left(y \gg \kappa^{-1}\right) \rightarrow \frac{2}{\kappa}\left[\alpha^{1 / 2} \ln \left(2 \alpha^{1 / 2}\right)+\alpha^{1 / 2} \frac{\kappa y}{2}-\alpha^{1 / 2}-\alpha^{1 / 2} \sinh ^{-1} \alpha^{1 / 2}+(\alpha+1)^{1 / 2}\right], \\
I_{1}^{\text {out }}(y \ll 1) \rightarrow-\frac{2}{3} \alpha^{1 / 2}+\alpha^{1 / 2} y+C,
\end{gathered}
$$

The term that depends on $y$ is the same in either limit, which confirms that $C$ is indeed a constant that can be determined by matching. Finally, a uniform approximation for the integral is obtained by combining the above expressions

$$
\begin{aligned}
I_{1}^{\text {app }}(y)= & I_{1}^{\text {in }}(y)+I_{1}^{\text {out }}(y)-I_{1}^{\text {match }}(y) \\
= & \frac{2}{\kappa}\left[\alpha^{1 / 2} \sinh ^{-1}\left(\alpha e^{\kappa y}\right)^{1 / 2}-\left(\alpha+e^{-\kappa y}\right)^{1 / 2}\right]_{0}^{y} \\
& -\frac{2}{3} \alpha^{1 / 2}\left[(1-y)^{3 / 2}-1+\frac{3}{2} y\right] .
\end{aligned}
$$

We compare in Table 2 this approximation with the exact values of the integral (evaluated numerically) for $\alpha=0.1$ and $\kappa=100,200$. For $\kappa=O(100)$, the maximum difference is no larger than $O\left(10^{-3}\right)$, which is practically insignificant. The larger the $\kappa$, the more accurate the approximation.

3.3 Case 2: $\alpha<0, \alpha+1>\beta, \tau_{m}>-\beta$

With an adverse pressure gradient, the stress distribution is non-monotonic. The stress changes abruptly near the wall, namely from a positive value at $y=0$ to a maximum 
Table 2: Comparison between the numerically evaluated exact values of the integral $I_{1}$ and the analytical approximation $I_{1}^{\text {app }}$ given by Eq. (28), where $\alpha=0.1$.

\begin{tabular}{c|cc|cc}
\hline \multirow{2}{*}{$y$} & \multicolumn{2}{|c|}{$\kappa=100$} & \multicolumn{2}{c}{$\kappa=200$} \\
\cline { 2 - 5 }$y$ & $I_{1}^{\text {exact }}$ & $I_{1}^{\text {app }}$ & $I_{1}^{\text {exact }}$ & $I_{1}^{\text {app }}$ \\
\hline 0.01 & $8.488 \times 10^{-3}$ & $8.484 \times 10^{-3}$ & $7.111 \times 10^{-3}$ & $7.107 \times 10^{-3}$ \\
0.05 & $2.533 \times 10^{-2}$ & $2.529 \times 10^{-2}$ & $2.051 \times 10^{-2}$ & $2.050 \times 10^{-2}$ \\
0.1 & $4.064 \times 10^{-2}$ & $4.060 \times 10^{-2}$ & $3.572 \times 10^{-2}$ & $3.571 \times 10^{-2}$ \\
0.5 & 0.1461 & 0.1461 & 0.1412 & 0.1412 \\
1.0 & 0.2206 & 0.2206 & 0.2157 & 0.2157 \\
\hline
\end{tabular}

negative value $\tau_{m}$ at $y=y_{m}$, where

$$
\tau_{m}=\alpha\left(1-y_{m}-\kappa^{-1}\right) \quad \text { and } \quad y_{m}=\kappa^{-1} \ln (-\kappa / \alpha) .
$$

The stress then changes gradually from this maximum negative value to zero at $y=1$.

The case shown in Fig. 2(c) is subject to the condition $\tau_{m}>-\beta$, by which the fluid is unsheared throughout the negative stress domain. Again, the fluid can be set into motion only if the condition given in Eq. (19) is satisfied. Under these conditions, there is only one yield surface, which is located within the EDL near the wall. The yield height $y_{0}$ is found numerically from

$$
y_{0}=-\kappa^{-1} \ln \left[\beta-\alpha\left(1-y_{0}\right)\right],
$$

where $y_{0} \approx-\kappa^{-1} \ln (\beta-\alpha)$ can be used as an initial value for solution by iteration.

In the sheared region $0 \leq y<y_{0}$, where the stress is positive, the velocity satisfying no slip at the wall has formally the same expression as that in Case 1, given in Eq. (22):

$$
u(y)=\alpha \kappa\left(y-\frac{1}{2} y^{2}\right)+\beta \kappa y+1-e^{-\kappa y}-2 \beta^{1 / 2} \kappa I_{2}(y),
$$

where $I_{2}$ is the following integral, which resembles $I_{1}$,

$$
I_{2}(y)=\int_{0}^{y}\left[\alpha(1-y)+e^{-\kappa y}\right]^{1 / 2} d y \text {. }
$$


Owing to the fact that $\alpha$ is negative in this case, the approximation derived for $I_{1}$ in Case 1 cannot be applied to $I_{2}$ here. Since the upper limit of the integral domain $y_{0} \ll 1$ is very small, a good approximation for $I_{2}$ is

$$
\begin{aligned}
I_{2}^{\text {app }}(y) & =\int_{0}^{y}\left[\alpha+e^{-\kappa y}\right]^{1 / 2} d y \\
& =\frac{2}{\kappa}\left[(-\alpha)^{1 / 2} \tan ^{-1}\left(-1-\frac{e^{-\kappa y}}{\alpha}\right)^{1 / 2}-\left(\alpha+e^{-\kappa y}\right)^{1 / 2}\right]_{0}^{y} .
\end{aligned}
$$

In the unsheared region $y_{0}<y \leq 1$, the plug flow velocity is $u_{p}=u\left(y_{0}\right)$. Like the base case, the plug flow extends across nearly the whole channel section. The adverse pressure gradient is not strong enough to cause any negative shear, so the velocity is always positive in this case.

\subsection{Case 3: $\alpha<0, \alpha+1>\beta, \tau_{m}<-\beta$}

In this case, shown in Fig. 2(d), there are multiple yielded and unyielded regions. First, the wall stress is positive and larger than the yield stress. This is the condition given in Eq. (19). Second, the maximum negative stress is also larger in magnitude than the yield stress: $\tau_{m}<-\beta$, where $\tau_{m}$ is given in Eq. (29). Consequently, there are three yield surfaces: two near the wall, and one far from the wall.

The three yield heights are determined iteratively by the following equations

$$
\begin{gathered}
y_{01}=-\kappa^{-1} \ln \left[\beta-\alpha\left(1-y_{01}\right)\right], \\
y_{02}=-\kappa^{-1} \ln \left[-\beta-\alpha\left(1-y_{02}\right)\right], \\
y_{03}=1+\left(\beta+e^{-\kappa y_{03}}\right) / \alpha,
\end{gathered}
$$

where $y_{01} \approx-\kappa^{-1} \ln (\beta-\alpha), y_{02} \approx-\kappa^{-1} \ln (-\beta-\alpha)$, and $y_{03} \approx 1+\beta / \alpha$ can be used as the initial values for the iteration.

In the first sheared region, $0 \leq y<y_{01}$, the velocity $u_{1}(y)$ can be determined using Eqs. (31)-(33) that are derived for Case 2. In the first unsheared region, $y_{01}<y<y_{02}$, the plug flow velocity is given by continuity of velocity $u_{p 1}=u_{1}\left(y_{01}\right)$. The velocity is always positive in these two regions. 
In the second sheared region, $y_{02}<y<y_{03}$, where the stress is negative, the velocity can be obtained as follows:

$$
\begin{aligned}
u_{2}(y)= & u_{p 1}+\alpha \kappa\left[y-y_{02}-\frac{1}{2}\left(y^{2}-y_{02}^{2}\right)\right] \\
& -\beta \kappa\left(y-y_{02}\right)+e^{-\kappa y_{02}}-e^{-\kappa y}+2 \beta^{1 / 2} \kappa I_{3}(y),
\end{aligned}
$$

where $I_{3}$ is an integral of the nonlinear coupling term

$$
I_{3}(y)=\int_{y_{02}}^{y}\left[-\alpha(1-y)-e^{-\kappa y}\right]^{1 / 2} d y \text {. }
$$

There exists no exact analytical expression for this integral. Again, in view of the boundary-layer structure of the integrand, we may seek a uniform approximation for $I_{3}$ using the boundary-layer theory. The procedure is the same as that we have described for the approximation of $I_{1}$ in Case 1. Without showing the intermediate steps, we give the final expression for the approximation of $I_{3}$ as follows:

$$
\begin{aligned}
I_{3}^{\text {app }}(y)= & -\frac{2}{3}(-\alpha)^{1 / 2}\left[(1-y)^{3 / 2}-\left(1-y_{02}\right)^{3 / 2}+\frac{3}{2}\left(1-y_{02}\right)^{1 / 2}\left(y-y_{02}\right)\right] \\
& +\frac{2 e^{-\kappa y_{02} / 2}}{\kappa}\left[\alpha^{\prime / 2} \tanh ^{-1}\left(1-\alpha^{\prime-1} e^{-\kappa\left(y-y_{02}\right)}\right)^{1 / 2}\right. \\
& \left.-\left(\alpha^{\prime}-e^{-\kappa\left(y-y_{02}\right)}\right)^{1 / 2}\right]_{y_{02}}^{y},
\end{aligned}
$$

where

$$
\alpha^{\prime}=-\alpha\left(1-y_{02}\right) e^{\kappa y_{02}}
$$

We have also checked the accuracy of this approximation by comparing it with some exact values of the integral. The accuracy is comparable to that of $I_{1}^{\text {app }}$. In the second unsheared region $y_{03}<y \leq 1$, the plug flow velocity is determined by continuity of velocity $u_{p 2}=u_{2}\left(y_{03}\right)$. The velocity in the second sheared region can be positive, zero, or negative, depending on $\alpha$. As a result, the net flow through the channel can be positive, zero, or negative in this case.

\subsection{Case 4: $\alpha<0,-\beta<1+\alpha<\beta, \tau_{m}<-\beta$}

In this case, as shown in Fig. 2(e), the wall stress is smaller in magnitude than the yield stress $-\beta<1+\alpha<\beta$. The maximum negative stress is, however, larger in magnitude than the yield stress: $\tau_{m}<-\beta$, where $\tau_{m}$ is given in Eq. (29). There are two 
yield surfaces, separating one sheared region from two unsheared regions. The two yield heights, denoted by $y_{02}$ and $y_{03}$, can be determined using Eqs. (35)-(36) as in Case 3.

In the first unsheared region $0 \leq y<y_{02}$, owing to no slip at the wall, the velocity is zero: $u_{p 1}=0$. In the sheared region $y_{02}<y<y_{03}$, the velocity $u(y)$ can be determined using Eqs. (37)-(40) that have been derived for $u_{2}(y)$ in Case 3. In the second unsheared region $y_{03}<y \leq 1$, the plug flow velocity is $u_{p 2}=u\left(y_{03}\right)$. The velocity is everywhere negative across the reduced section $y_{02} \leq y \leq 1$.

\subsection{Case 5: $\alpha<0,1+\alpha<-\beta$}

In this case, as shown in Fig. 2(f), the wall stress is negative and is larger in magnitude than the yield stress $1+\alpha<-\beta$. There is only one yield surface located far from the wall. The yield height, denoted by $y_{03}$, can be determined using Eq. (36) as in Case 3. In the sheared region $0 \leq y<y_{03}$, the velocity $u(y)$ can be determined using Eqs. (37)-(40), where $u_{p 1}=0$ and $y_{02}=0$. In the unsheared region $y_{03}<y \leq 1$, the plug flow velocity is $u_{p}=u\left(y_{03}\right)$. The velocity is negative across the entire channel.

\section{Discussion}

The present problem depends on three parameters: the normalized pressure gradient $\alpha$, the normalized yield stress $\beta$, and the normalized Debye parameter $\kappa$. In the following, we shall examine results to find out how the EO flow may be affected by the yield stress of a Casson fluid under a favorable or adverse pressure gradient. A sufficiently large Debye parameter $\kappa=100$, for a very thin EDL, is used in all our calculations. Let us first have an order of magnitude estimate about the yield stress parameter $\beta$, which is defined in Eq. (12). Consider blood in particular. When modeled as a Casson fluid, blood has a yield stress that depends strongly on the hematocrit (the volume fraction of red blood cells) and the fibrinogen (a protein involved in clotting) concentration. For normal human blood at a hematocrit of 45\%, the yield stress lies between 1 and $6 \mathrm{mPa}$ [31]. A typical value commonly adopted in the literature for blood flow analysis is 4 or $5 \mathrm{mPa}$ [32-34]. Such a yield stress is much smaller in magnitude than the normal 
blood pressure and is often deemed to be of little physiological significance. We have here normalized the yield stress $\tau_{0}$ by the electric wall stress $\tau_{E}=-\epsilon \kappa \zeta E_{x}$. Hence, for $\epsilon \approx 5 \times 10^{-10} \mathrm{C} /(\mathrm{Vm})$ (permittivity of blood), $E_{x} \approx 10 \mathrm{~V} / \mathrm{cm}, \zeta \approx-10 \mathrm{mV}$, and $\kappa^{-1} \approx 10 \mathrm{~nm}$ (based on an ionic strength of $1 \mathrm{mM}[3]$ ), we may get $\tau_{E} \approx 500 \mathrm{mPa}$, and therefore $\beta=\tau_{0} / \tau_{E}=O(0.01)$. This calculation shows that the parameter is indeed very small, but the effect of a small parameter in the present problem is not necessarily small. As has been shown earlier and will be further shown below, a normalized yield stress of the order 0.01 can already cause appreciable effect on EO flow in a microchannel. Our model is of course applicable to other Casson fluids than blood. To make our discussion more general, values of $\beta$ ranging from zero to unity are considered, as presented below.

Figure 3 shows the flow rate $q=\int_{0}^{1} u d y$ as a function of the normalized pressure gradient $\alpha$, for normalized yield stress $\beta=0,0.01,0.1$, where $\kappa=100$. The flow rate has been evaluated numerically by Simpson's rule. In the absence of yield stress $\beta=0$, corresponding to Newtonian fluid, the flow rate varies linearly with the pressure gradient, as expected. A small yield stress $\beta=0.01$ is, however, sufficient to cause appreciable departure from the linear limit. There is a range in the neighborhood of $\alpha=0$, where the flow rate varies slowly with $\alpha$. This happens when the magnitude of $\alpha$ is so small that the stress outside the EDL is smaller in magnitude than the yield stress, corresponding to the Base Case, Case 1(a), or Case 2 of the stress distribution. Hence, the stress outside the EDL, which is due to the pressure gradient, has no effect on the flow. It is entirely a plug flow outside the EDL, and the flow rate only weakly depends on $\alpha$ within this range of $\alpha$. This range of $\alpha$ is larger for larger $\beta$. Even outside this range, the flow rate will increase with $\alpha$ at a rate smaller than that of the linear limit. Hence, the decreasing effect of the yield stress on the flow intensifies as the pressure gradient increases in magnitude.

Under Case 3 of the stress distribution, for given $\beta$ and $\kappa$, there exists a unique value of $\alpha$ for which the net flow is zero. We show in the inset of Fig. 3 these particular values of $\alpha$ for the admissible range of $\beta$. One can show that, for $\kappa=100$, the maximum $\beta$ is 0.4838 for a possible Case 3 stress distribution. When $\beta$ exceeds this value, there exists no fluid in motion that will correspond to a zero net flow; only fluid at rest will do.

For illustration, some velocity profiles for $\beta=0$ and $\beta=0.1$ are shown in Figs. 4(a,b), 
respectively. Those for the Newtonian limit $\beta=0$ serve as a basis of comparison. The difference between the Newtonian and the Casson velocity profiles is indeed very large. Under the same $\alpha$, the velocities may differ from each other in the magnitude, the sign, as well as the shape of the profile.

In Fig. 4(b), the heights of the yield surfaces, $y_{0}$, are marked by open circles on the velocity profiles. We also denote by the numbers in the brackets the types of stress distribution associated with these profiles. As noted above, for a small magnitude of $\alpha$, the fluid is completely unsheared away from the EDL. As $\alpha$ increases in magnitude, the sheared region also increases in size, in which the velocity is largely parabolic outside the EDL. There are three yield surfaces for a Case 3 stress distribution: two near the wall, and one far from the wall. The unsheared region in the EDL, if any, is very small in size, and can practically be ignored. The net flow is zero when $\alpha=-0.18367$ for $\beta=0.1$ and $\kappa=100$. In this case, the flow is positive near the wall, but is a negative plug flow in the core of the channel.

We further show in Fig. 5 how the flow is sensitively affected by the yield stress. We have shown earlier that in the Base Case $\alpha=0$, the flow is decreased by some $12 \%$ for $\beta=10^{-3}$, and $32 \%$ for $\beta=10^{-2}$. This decreasing effect due to the yield stress will be enhanced by the presence of a pressure gradient, whether favorable or adverse. Our results show that, for $\alpha=0.1$, the flow is decreased by $19 \%$ for $\beta=10^{-3}$, and $51 \%$ for $\beta=10^{-2}$. The effect is even more dramatic under an adverse pressure gradient. For $\alpha=-0.1$, the flow is decreased in magnitude by $27 \%$ for $\beta=10^{-3}$, and $71 \%$ for $\beta=10^{-2}$. In fact, for the results shown in Fig. 5 , it is within the range $10^{-4}<\beta<10^{-1}$ where the flow is sensitively affected by the yield stress.

For further illustration, we show some velocity profiles in Figs. 6(a,b), for $\alpha=0.1$ and $\alpha=-0.1$, respectively. Increasing the yield stress will reduce the shear rate, or the velocity gradient, in the sheared region. In the case of $\beta=0.01$, the plug flow region remains small in extent, but the velocity in the sheared region is already much reduced in magnitude. In particular, the net flow becomes zero when $\beta=0.03066$ for $\alpha=-0.1$ and $\kappa=100$. For a larger yield stress $\beta=0.1$, the flow profile diminishes to become a positive plug flow outside the EDL, whether $\alpha=0.1$ or $\alpha=-0.1$. As already explained above, this happens when the stress induced by the pressure gradient 
is smaller in magnitude than the yield stress. The flow has then a weak dependence on $\alpha$ for such a pressure gradient. In summary, the yield stress is to play two roles here. First, it introduces a plug flow region. Second, it reduces the shear rate, and therefore the magnitude of velocity, in the sheared region. For small $\beta$, while the first effect remains small, the second effect can already be influential. For large $\beta$, both effects are important, thereby resulting in a full plug flow but of a diminished magnitude outside the EDL.

\section{Concluding remarks}

In this paper, we have presented analytical solutions for one-dimensional steady flow of a Casson fluid, under the combined action of pressure gradient and electroosmotic (EO) pumping, through a parallel-plate channel. The problem is simplified by the assumptions of very small potentials (or the Debye-Hückel approximation) and a very thin electric double layer (EDL) compared with the channel height. The flow analysis starts with locating the yield surfaces in order to partition the flow section into sheared and unsheared regions. Six possible cases of the distribution of stress relative to the yield stress are identified. Depending on the magnitude as well as the sign of the pressure gradient, it is possible to have one or multiple yield surfaces in the flow section. The velocity in the sheared region can be straightforwardly obtained by integrating the Cauchy momentum equation. Using the boundary-layer theory, we have deduced uniform approximations for the integrals of the nonlinear coupling terms of the two forcings. All velocities in the present problem are thereby analytically expressed.

Even a small value of the Casson yield stress, characteristic of that of blood, can considerably decrease the rate of EO flow in a microchannel. The Smoluchowski velocity is found to be sensitively affected by the Casson yield stress. This velocity, owing to nonlinear rheology, can no longer be used as an EO slip boundary condition for flow of a non-Newtonian fluid in general. The presence of a pressure gradient, whether favorable or adverse, will enhance the decreasing effect of the yield stress. The yield stress is to introduce a plug-flow region, the size of which increases as the yield stress increases. More dramatically, the yield stress will diminish the shear rate in a sheared region. 
It is worth extending the problem to include some more complicated effects, such as the skimming layer near the wall, high potentials without the Debye-Hückel approximation, two-dimensional or unsteady flows, and so on. It is also worth comparing EO flow of Casson fluid with that of other viscoplastic materials, such as Bingham plastic and Herschel-Bulkley fluid.

\section{Acknowledgments}

The work was financially supported by the Research Grants Council of the Hong Kong Special Administrative Region, China, through Project HKU 715510E.

\section{References}

[1] H.A. Stone, A.D. Stroock, A. Ajdari, Engineering flows in small devices: microfluidics toward a lab-on-a-chip, Annu. Rev. Fluid Mech. 36 (2004) 381-411.

[2] B.J. Kirby, Micro- and Nanoscale Fluid Mechanics, Cambridge University Press, Cambridge, 2010.

[3] S. Das, S. Chakraborty, Analytical solutions for velocity, temperature and concentration distribution in electroosmotic microchannel flows of a non-Newtonian bio-fluid, Anal. Chim. Acta 559 (2006) 15-24.

[4] W.B. Zimmerman, J.M. Rees, T.J. Craven, Rheometry of non-Newtonian electrokinetic flow in a microchannel T-junction, Microfluid. Nanofluid. 2 (2006) 481492.

[5] S. Chakraborty, Electroosmotically driven capillary transport of typical nonNewtonian biofluids in rectangular microchannels, Anal. Chim. Acta 605 (2007) $175-184$.

[6] C.L.A. Berli, M.L. Olivares, Electrokinetic flow of non-Newtonian fluids in microchannels, J. Colloid Interface Sci. 320 (2008) 582-589. 
[7] C. Zhao, E. Zholkovskij, J. Masliyah, C. Yang, Analysis of electroosmotic flow of power-law fluids in a slit microchannel, J. Colloid Interface Sci. 326 (2008) $503-510$.

[8] M.L. Olivares, L. Vera-Candioti, C.L.A. Berli, The EOF of polymer solutions, Electrophoresis 30 (2009) 921-929.

[9] C. Zhao, C. Yang, Analysis of power-law fluid flow in a microchannel with electrokinetic effects, Int. J. Emerg. Multi. Fluid Sci. 1 (2009) 37-52.

[10] C. Zhao, C. Yang, Nonlinear Smoluchowski velocity for electroosmosis of powerlaw fluids over a surface with arbitrary zeta potentials, Electrophoresis 31 (2010) 973-979.

[11] C. Zhao, C. Yang, An exact solution for electroosmosis of non-Newtonian fluids in microchannels, J. Non-Newtonian Fluid Mech. 166 (2011) 1076-1079.

[12] C.L.A. Berli, Output pressure and efficiency of electrokinetic pumping of nonNewtonian fluids, Microfluid. Nanofluid. 8 (2010) 197-207.

[13] N. Vasu, S. De, Electroviscous effects in purely pressure driven flow and stationary plane analysis in electroosmotic flow of power-law fluids in a slit microchannel, Int. J. Eng. Sci. 48 (2010) 1641-1658.

[14] A. Sadeghi, M. Fattahi, M.H. Saidi, An approximate analytical solution for electroosmotic flow of power-law fluids in a planar microchannel, J. Heat Trans. 133 (2011) 091701.

[15] R.P. Bharti, D.J.E. Harvie, M.R. Davidson, Electroviscous effects in steady fully developed flow of a power-law liquid through a cylindrical microchannel, Int. J. Heat Fluid Flow 30 (2009) 804-811.

[16] G.H. Tang, X.F. Li, Y.L. He, W.Q. Tao, Electroosmotic flow of non-Newtonian fluid in microchannels, J. Non-Newtonian Fluid Mech. 157 (2009) 133-137.

[17] N. Vasu, S. De, Electroosmotic flow of power-law fluids at high zeta potentials, Colloids and Surfaces A: Physicochem. Eng. Aspects 368 (2010) 44-52. 
[18] A. Babaie, A. Sadeghi, M.H. Saidi, Combined electroosmotically and pressure driven flow of power-law fluids in a slit microchannel, J. Non-Newtonian Fluid Mech. 166 (2011) 792-798.

[19] M. Hadigol, R. Nosrati, M. Raisee, Numerical analysis of mixed electroosmotic/pressure driven flow of power-law fluids in microchannels and micropumps, Colloids and Surfaces A: Physicochem. Eng. Aspects 374 (2011) 142-153.

[20] C.C. Cho, C.L. Chen, C.K. Chen, Electrokinetically-driven non-Newtonian fluid flow in rough microchannel with complex-wavy surface, J. Non-Newtonian Fluid Mech. 173-174 (2012) 13-20.

[21] C.C. Cho, C.L. Chen, C.K. Chen, Flow characteristics and mixing performance of electrokinetically driven non-Newtonian fluid in contraction-expansion microchannel, Rheol. Acta 51 (2012) 925-935.

[22] S.Y. Deng, Y.J. Jian, Y.H. Bi, L. Chang, H.J. Wang, Q.S. Liu, Unsteady electroosmotic flow of power-law fluid in a rectangular microchannel, Mech. Res. Comm. 39 (2012) 9-14.

[23] M. Shamshiri, R. Khazaeli, M. Ashrafizaadeh, S. Mortazavi, Electroviscous and thermal effects on non-Newtonian liquid flows through microchannels, J. NonNewtonian Fluid Mech. 173-174 (2012) 1-12.

[24] M.A. Vakili, A. Sadeghi, M.H. Saidi, A.A. Mozafari, Electrokinetically driven fluidic transport of power-law fluids in rectangular microchannels, Colloids and Surfaces A: Physicochem. Eng. Aspects 414 (2012) 440-456.

[25] G.H. Tang, P.X. Ye, W.Q. Tao, Pressure-driven and electroosmotic nonNewtonian flows through microporous media via lattice Boltzmann method, J. Non-Newtonian Fluid Mech. 165 (2010) 1536-1542.

[26] C. Zhao, C. Yang, Electro-osmotic mobility of non-Newtonian fluids, Biomicrofluidics 5 (2011) 014110.

[27] R.B. Bird, G.C. Dai, B.J. Yarusso, The rheology and flow of viscoplastic materials, Rev. Chem. Eng. 1 (1983) 1-70. 
[28] M. Liu, J. Yang, Electrokinetic effect of the endothelial glycocalyx layer on twophase blood flow in small blood vessels, Microvascular Res. 78 (2009) 14-19.

[29] M. Liu, Y. Liu, Q. Guo, J. Yang, Modeling of electroosmotic pumping of nonconducting liquids and biofluids by a two-phase flow method, J. Electroanal. Chem. 636 (2009) 86-92.

[30] N. Casson, A flow equation for pigment-oil suspensions of the printing ink type, in Rheology of Disperse Systems, C.C. Mill (ed), 84-104, Pergamon, London, 1959.

[31] R.L. Whitmore, Rheology of the Circulation, Pergamon, London, 1968.

[32] E.W. Merrill, Rheology of blood, Physiol. Rev. 49 (1969) 863-888.

[33] C.R. Ethier, C.A. Simmons, Introductory Biomechanics, Cambridge University Press, Cambridge, 2007.

[34] R.L. Fournier, Basic Transport Phenomena in Biomedical Engineering, Taylor \& Francis, NY, 2007. 

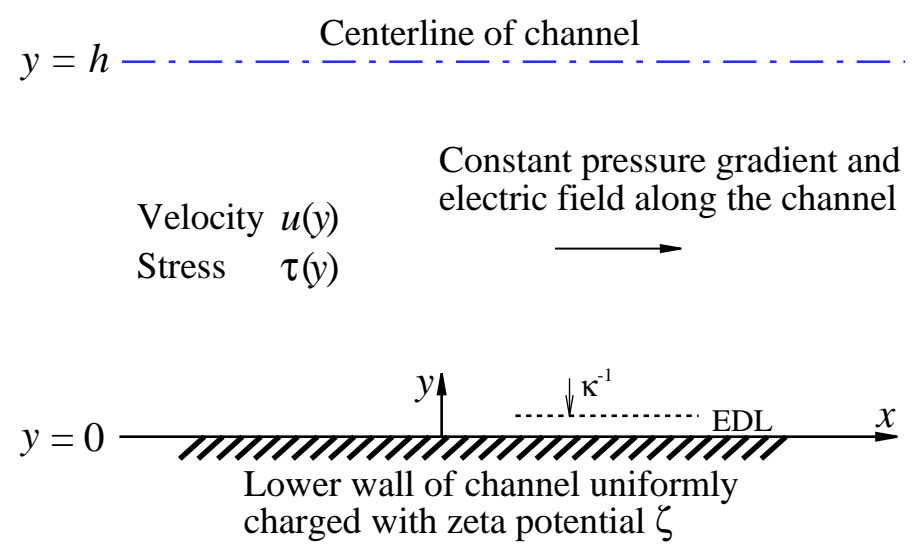

Figure 1: Definition sketch of the problem: one-dimensional flow of velocity $u(y)$ and shear stress $\tau(y)$, forced by a constant pressure gradient and electric field, through a slit channel of height $2 h$. The wall is uniformly charged with a constant zeta potential $\zeta$. The electric double layer (EDL) is much thinner than the channel height $\kappa h \gg 1$. 

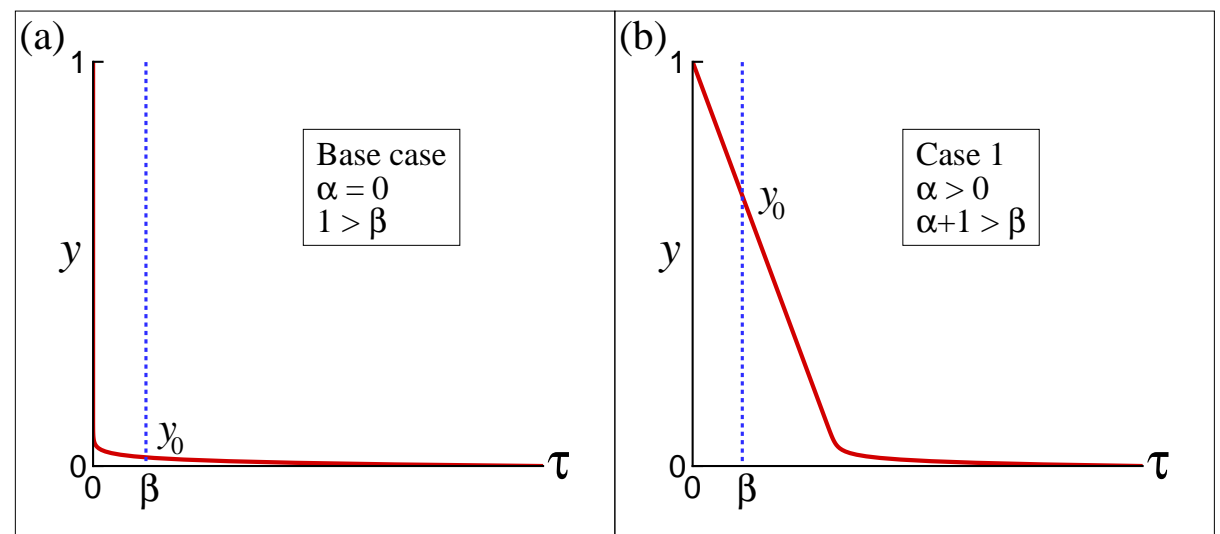

(c)

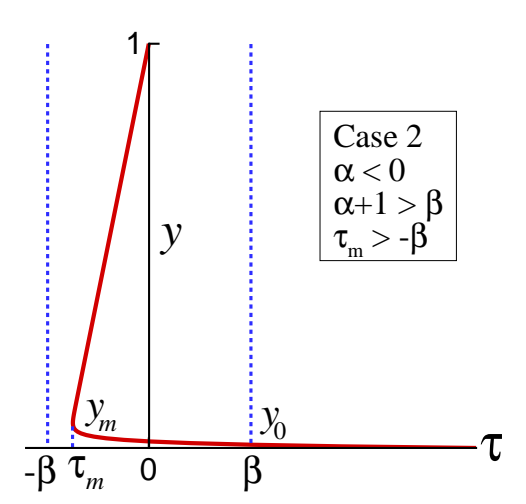

(d)

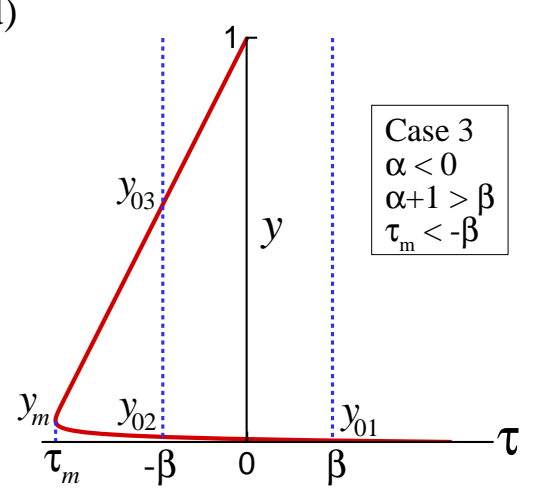

(e)

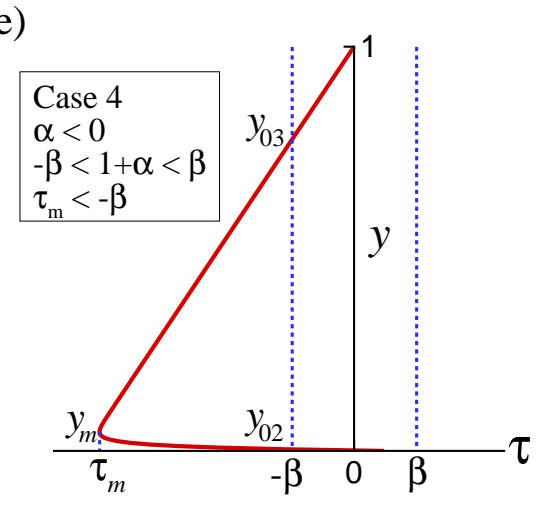

(f)

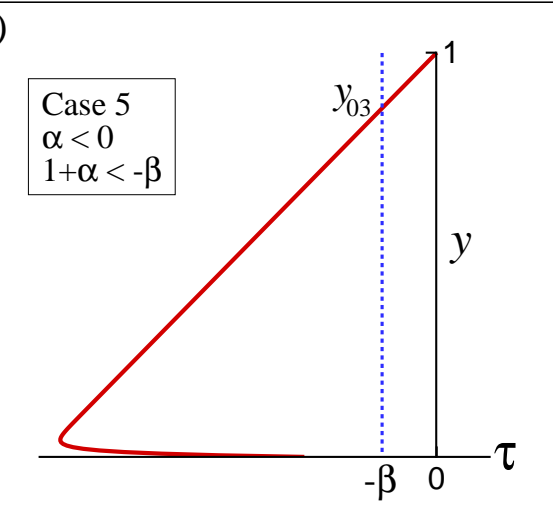

Figure 2: Definition sketch of six possible cases of distribution of the stress $\tau(y)$ as a result of different combinations of the pressure and electric forcings, where $\alpha$ is the normalized pressure gradient, $\beta$ is the normalized yield stress, $y_{0}, y_{01}, y_{02}, y_{03}$ are the heights of the yield surfaces where $|\tau|=\beta$. The maximum negative stress $\tau_{m}$ is at the height $y_{m}$. The stress distributions will occur under the conditions given in the text boxes. The fluid is sheared in the regions where $|\tau|>\beta$, and is unsheared in the regions where $|\tau|<\beta$. 


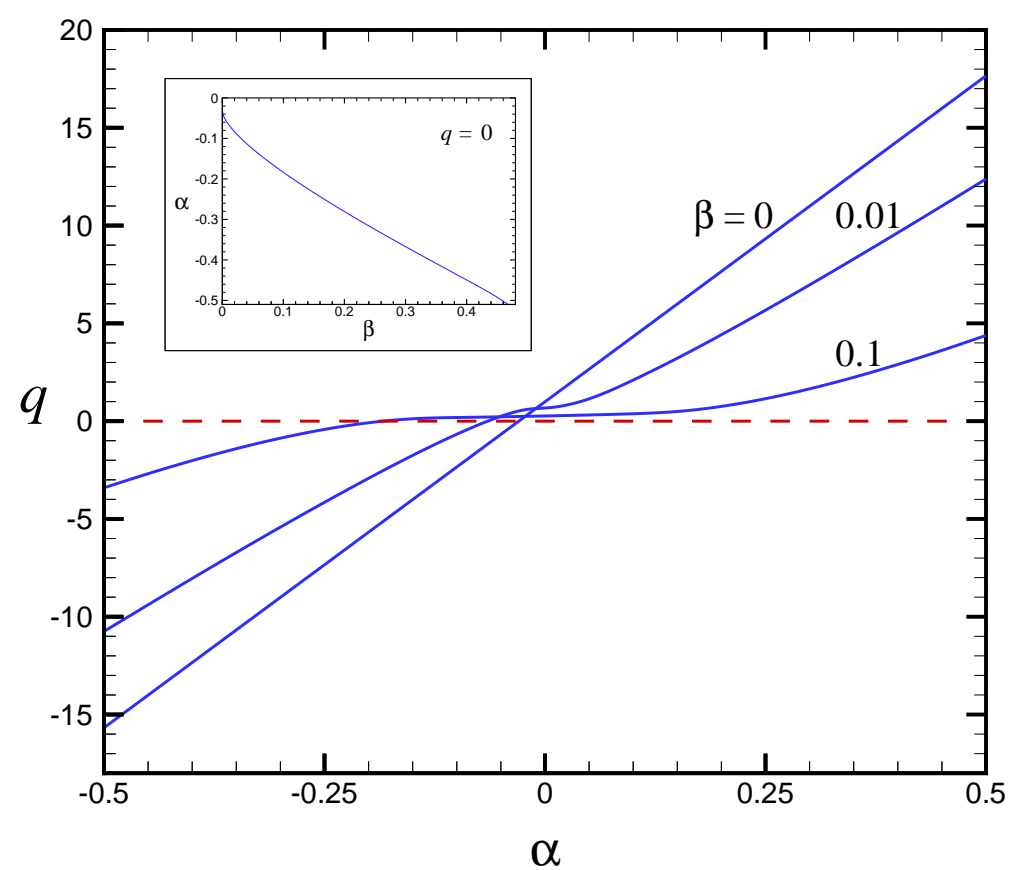

Figure 3: The flow rate $q$ as a function of the normalized pressure gradient $\alpha$, for the normalized yield stress $\beta=0,0.01,0.1$. Inset: particular values of $\alpha$ and $\beta$ for which $q=0$. 

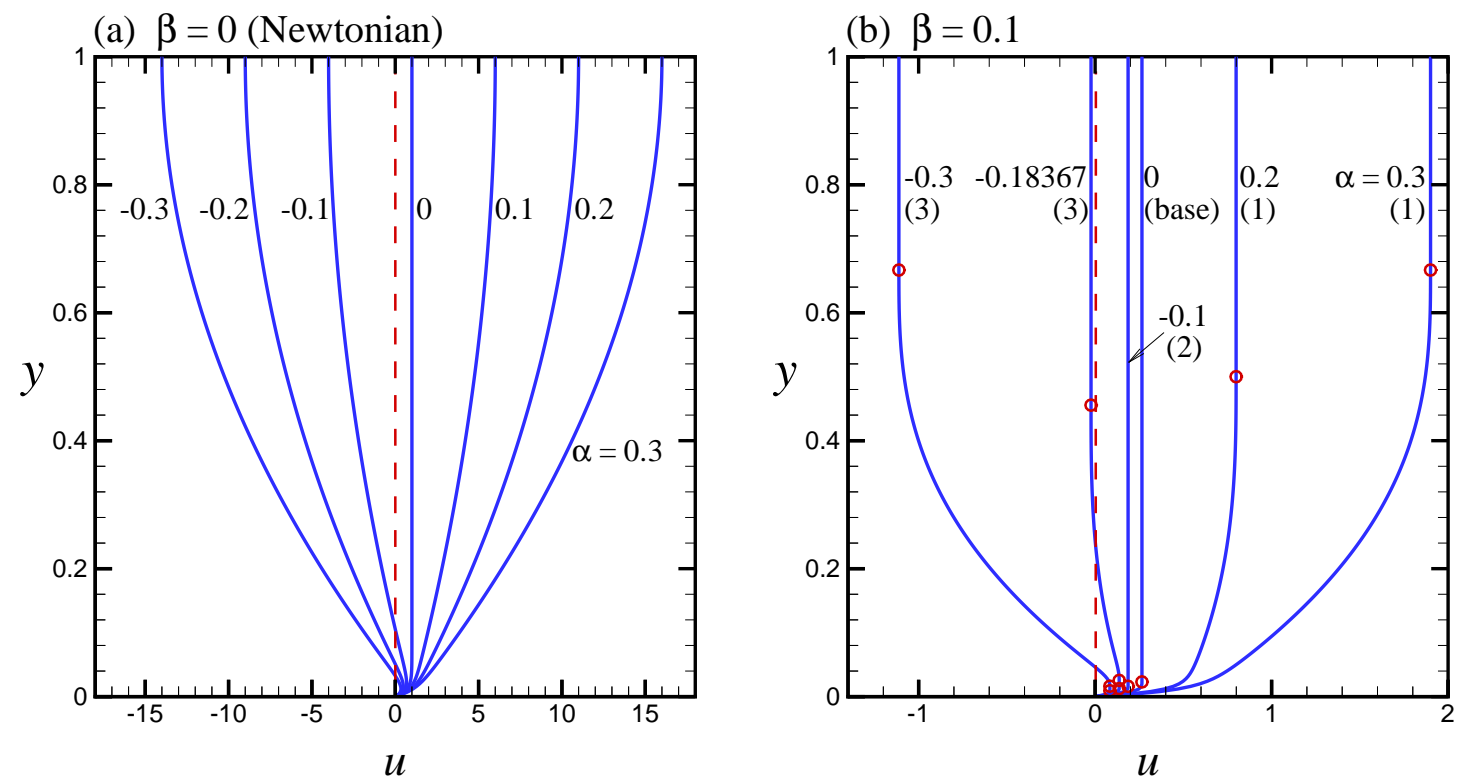

Figure 4: Velocity profiles $u(y)$ for various $\alpha$, where (a) $\beta=0$ (Newtonian), and (b) $\beta=0.1$. The open circles are the places where the stress is equal in magnitude to the yield stress, and the numbers in brackets denote the cases of stress distribution associated with the velocity profiles. 


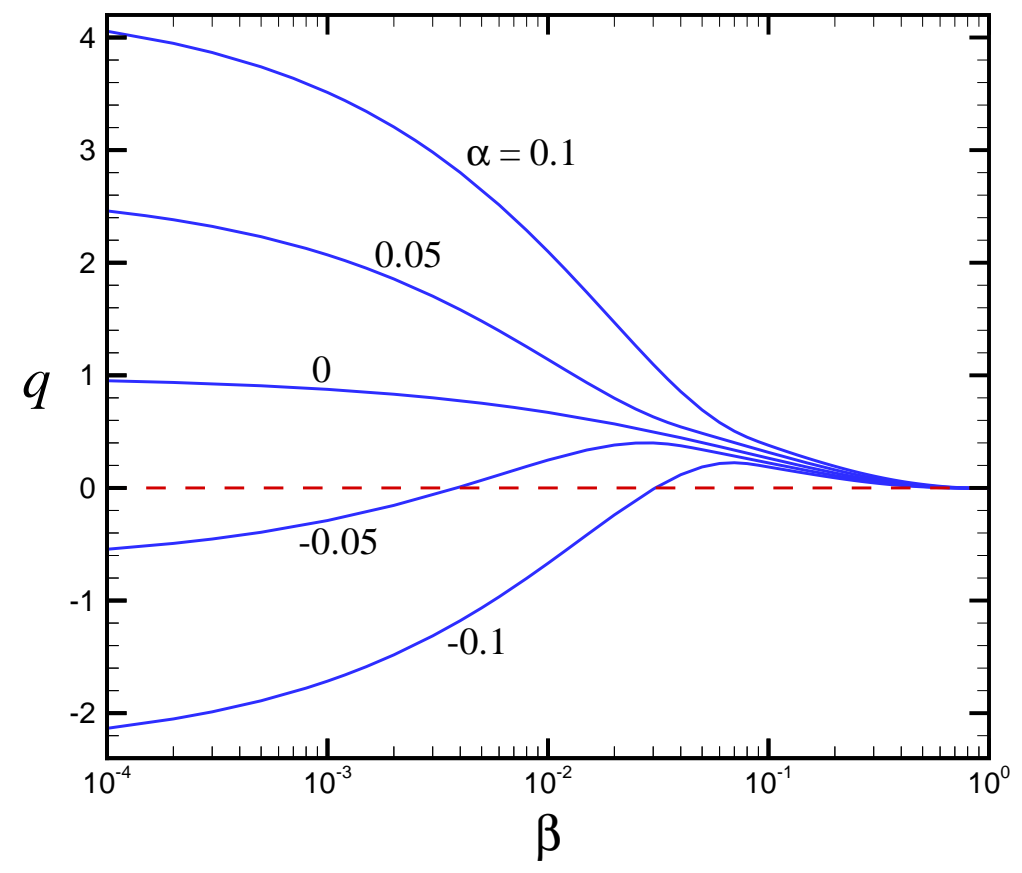

Figure 5: The flow rate $q$ as a function of the normalized yield stress $\beta$, for the normalized pressure gradient $\alpha=0.1,0.05,0,-0.05,-0.1$. 

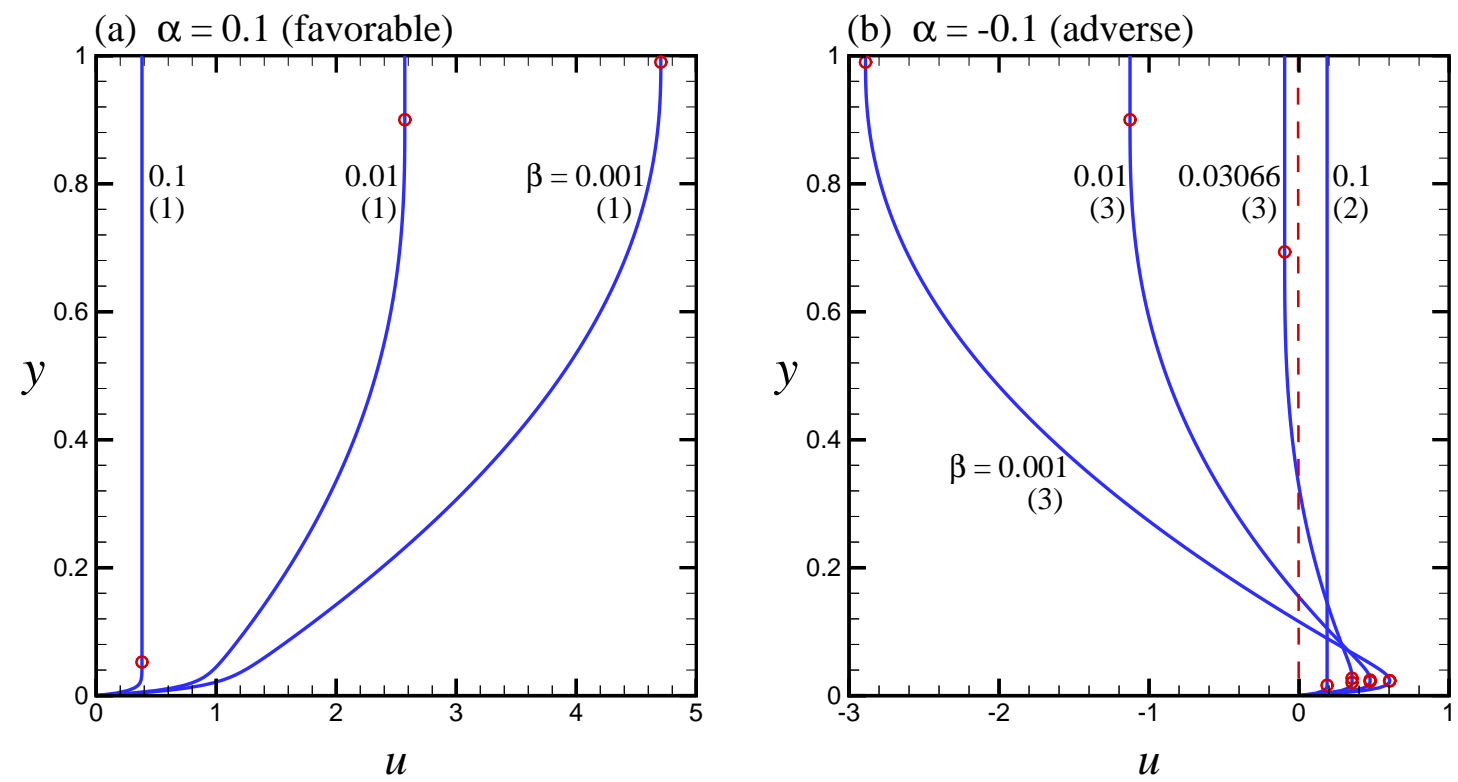

Figure 6: Velocity profiles $u(y)$ for various $\beta$, where (a) $\alpha=0.1$ (favorable), and (b) $\alpha=$ -0.1 (adverse). The open circles are the places where the stress is equal in magnitude to the yield stress, and the numbers in brackets denote the cases of stress distribution associated with the velocity profiles. 